\title{
Suscetibilidade de bagas de genótipos de videira pela infestação por Drosophila suzukii (Diptera: Drosophilidae)
}

\author{
Felipe Andreazza(1), Cléber Antonio Baronio(2), Marcos Botton(2), Ricardo Alexandre Valgas ${ }^{(3)}$, \\ Patrícia Silva Ritschel(2), João Dimas Garcia Maia( ${ }^{(2)}$ e Dori Edson Nava(3)
}

\begin{abstract}
(1)Universidade Federal de Pelotas, Campus Universitário, s/no, Caixa Postal 354, CEP 96160-000 Capão do Leão, RS, Brasil. E-mail: andreazzafelipe@yahoo.com.br (2)Embrapa Uva e Vinho, Rua Livramento, no 515, Caixa Postal 130, CEP $95700-000$ Bento Gonçalves, RS, Brasil. E-mail: cleber.baronio@hotmail.com, marcos.botton@embrapa.br, patricia.ritschel@embrapa.br, joao.maia@embrapa.br (3)Embrapa Clima Temperado, BR-392, Km 78, Caixa Postal 403, CEP 96010-971 Pelotas, RS, Brasil. E-mail: ricardo.valgas@embrapa.br, dori.edson-nava@embrapa.br
\end{abstract}

Resumo - O objetivo deste trabalho foi avaliar a suscetibilidade de genótipos de videira à drosófila-da-asamanchada, Drosophila suzukii (Diptera: Drosophilidae), em bagas de uva intactas e em bagas infestadas após a ocorrência de puncturas, causadas pela oviposição da mosca-das-frutas sul-americana [Anastrepha fraterculus (Diptera: Tephritidae)] ou por danos mecânicos (alfinetes). Os experimentos foram realizados em laboratório, a $22 \pm 1{ }^{\circ} \mathrm{C}$, umidade relativa de $65 \pm 10 \%$ e fotófase de 12 horas. A suscetibilidade foi avaliada para 18 genótipos de videira, em bagas intactas submetidas às fêmeas de $D$. suzukii. O potencial de interação foi verificado em bagas de uva 'Italia', cuja epiderme foi danificada por puncturas de A. fraterculus ou por alfinete, em comparação a frutos de morango 'Albion'. As cultivares de Vitis labrusca 'Niagara Rosada' e 'Concord' não foram infestadas por $D$. suzukii, e cinco dos oito genótipos que foram infestados são cultivares melhoradas. A infestação de D. suzukii em bagas de uva 'Italia' com danos mecânicos, feitos com um alfinete ou pela oviposição de $A$. fraterculus, foi semelhante à de bagas íntegras. Há baixa adequação hospedeira de videiras a D. suzukii, mesmo com a presença de danos. As cultivares 'Benitaka', 'BRS Vitória' e 'BRS Morena' são as mais suscetíveis a D. suzukii.

Termos para indexação: Anastrepha fraterculus, Vitis labrusca, Vitis vinifera, drosófila-da-asa-manchada, mosca-das-frutas.

\section{Susceptibility of grape vine genotypes by the infestation of Drosophila suzukii (Diptera: Drosophilidae)}

\begin{abstract}
The objective of this work was to evaluate the susceptibility of vine genotypes to the spotted wing drosophila, Drosophila suzukii (Diptera: Drosophilidae), on uninjured grapes and on infested grapes after the occurrence of puncture sites, caused either by the oviposition of South American fruit fly [Anastrepha fraterculus (Diptera: Tephritidae)] or by mechanical damage (pins). The experiments were carried out in laboratory at $22 \pm 1{ }^{\circ} \mathrm{C}, 65 \pm 10 \%$ relative humidity, and 12 -hour photophase. Susceptibility was evaluated on intact grapes of 18 genotypes subjected to $D$. suzukii females. The interaction potential was assessed on 'Italia' grapes, whose epidermis was damaged either by punctures caused by A. fraterculus or by pins, in comparison to 'Albion' strawberries. The 'Niagara Rosada' and 'Concord' Vitis labrusca cultivars were not infested by D. suzukii, and five out of the eight infested genotypes are improved varieties. Infestation of $D$. suzukii on 'Italia' grapes, with mechanical damage made by a pin or by $A$. fraterculus oviposition, was similar to that on undamaged grapes. There is a low suitability of host vines to $D$. suzukii, even with the presence of previous injuries. The 'Benitaka', 'BRS Vitória', and 'BRS Morena' vine cultivars are the most susceptible genotypes to D. suzukii.
\end{abstract}

Index terms: Anastrepha fraterculus, Vitis labrusca, Vitis vinifera, spotted wing drosophila, fruit fly.

\section{Introdução}

Drosophila suzukii (Matsumura, 1931) (Diptera: Drosophilidae), popularmente conhecida por drosófila-da-asa-manchada, é uma praga polífaga originária do Japão que se encontra atualmente em expansãomundial(Burrack etal.,2013; Cinietal., 2014). No Brasil, foi recentemente identificada em armadilhas instaladas em reservas florestais, nos estados do Rio Grande do Sul, Santa Catarina (Deprá et al., 2014) e 
São Paulo (Vilela \& Mori, 2014), tendo causado danos econômicos em morangueiro (Fragaria x ananassa Duch.) (Santos, 2014). Esta praga tem capacidade de infestar e causar danos em frutíferas com epiderme fina, principalmente as do grupo das pequenas frutas, como morango, amora-preta (Rubus sp.), framboesa (Rubus idaeus L.), mirtilo [Vaccinium ashei (Reade)] e cereja (Prunus sp.) (Cini et al., 2012; Santos, 2014). Contudo, recentes relatos sobre danos em cultivares de videira (Vitis spp.), com bagas de epiderme de cor rosada, nas regiões de Quebec, Canadá, e em Michigan, nos Estados Unidos (Saguez et al., 2013; van Timmeren \& Isaacs, 2014) têm preocupado a cadeia produtiva de videiras no Sul do Brasil, onde a praga está presente (Deprá et al., 2014; Santos, 2014). Outro fator que poderá favorecer a infestação pela $D$. suzukii, na região Sul do Brasil, é o fato de que a videira é cultivada em pequenas propriedades onde há uma diversidade de frutos que são hospedeiros deste inseto. Além disso, na Serra Gaúcha, principal polo produtor de uvas para processamento do país (Camargo et al., 2011), a presença de mata-atlântica com hospedeiros nativos da D. suzukii aumenta a preocupação de técnicos e produtores quanto ao potencial da espécie em causar danos ao cultivo.

Recentemente, verificou-se que espécies do gênero Vitis são menos preferidas para oviposição pela $D$. suzukii do que o morangueiro (Bellamy et al., 2013). Em estudo de suscetibilidade de uvas à $D$. suzukii, constatou-se que mesmo quando há oviposição em bagas danificadas, não ocorre a emergência dos insetos, e que cultivares para vinho de Vitis labrusca ('Concord') e Vitis vinifera ('Merlot' e 'Riesling') podem não apresentar diferença quanto à suscetibilidade ao ataque por esse inseto (Maiguashca et al., 2010). São necessários estudos de suscetibilidade que avaliem uma gama maior de cultivares $V$. vinifera $\mathrm{e}$ V. labrusca e cultivares provenientes de programas de melhoramento, para se conhecer o real potencial de dano desta nova praga na viticultura brasileira. Além disso, estas pesquisas poderão permitir a identificação de genótipos resistentes à $D$. suzukii.

No Brasil, a viticultura é diversificada em relação a cultivares e pragas que ocorrem nos vinhedos, merecendo destaque a mosca-das-frutas sul-americana, A. fraterculus (Wiedemann, 1830) (Diptera: Tephritidae). Os danos causados por esta mosca em videira estão relacionados à queda de bagas (Zart et al., 2011) e, também, ao transporte e à inoculação de patógenos como Cladosporium spp., Botrytis cinerea Pers., Colletotrichum spp. e Penicillium spp., que são favorecidos por puncturas para oviposição feitas pela mosca-das-frutas sul-americana (Machota Junior et al., 2013). Assim, é importante, também, saber se os danos causados pela mosca-das-frutas sul-americana, durante a oviposição, pode favorecer a infestação pela D. suzukii.

O objetivo deste trabalho foi avaliar a suscetibilidade de genótipos de videira à drosófila-da-asa-manchada [Drosophila suzukii (Diptera: Drosophilidae)], em bagas de uva intactas, e em bagas infestadas após a ocorrência de puncturas, causadas pela oviposição da mosca-das-frutas sul-americana [Anastrepha fraterculus (Diptera: Tephritidae)] e por danos mecânicos (alfinetes).

\section{Material e Métodos}

O trabalho foi realizado no Laboratório de Entomologia da Embrapa Uva e Vinho, em Bento Gonçalves, $\mathrm{RS}$, a $20^{\circ} 09^{\prime} 50^{\prime \prime} \mathrm{S}$ e $51^{\circ} 31^{\prime} 51^{\prime \prime} \mathrm{W}$, em fevereiro de 2015 , em condições controladas de temperatura a $22 \pm 1^{\circ} \mathrm{C}$, umidade relativa do ar a $65 \pm 10 \%$ e fotófase de 12 horas. Os adultos de D. suzukii, utilizados nos experimentos, foram obtidos da criação mantida no Laboratório de Entomologia, da Embrapa Clima Temperado, Pelotas, RS, a $31^{\circ} 40^{\prime} 47^{\prime \prime} \mathrm{S}$ e $52^{\circ} 26^{\prime} 21^{\prime \prime} \mathrm{W}$.

$\mathrm{Na}$ determinação da suscetibilidade de genótipos de videira à $D$. suzukii, bagas de 18 genótipos foram utilizadas, das quais três cultivares de $V$. labrusca ('Concord', 'Isabel' e 'Niagara Rosada'), sete cultivares de $V$. vinifera ('Benitaka', 'Cabernet Sauvignon', 'Italia', 'Itália Muscat', 'Moscato Bianco', 'Redglobe' e 'Italia Rubi'), cinco híbridos de variedades do programa de melhoramento da Embrapa Uva e Vinho ('BRS Isis', 'BRS Morena', 'BRS Núbia', 'BRS Vitória' e 'BRS Lorena') e três seleções avançadas do programa de melhoramento da Embrapa (CNPUV 1060-4, CNPUV 1097-258 e Seleção 20). Cachos destes genótipos foram obtidos de vinhedos localizados em áreas experimentais, sem aplicação de inseticidas. Na primeira quinzena de fevereiro de 2015, os frutos foram colhidos com as ráquis e acondicionados em câmara refrigerada a $0^{\circ} \mathrm{C}$, por cinco dias, quando foram realizados os testes.

Após a colheita, para cada genótipo, determinaramse as seguintes variáveis: resistência da epiderme à 
penetração, por meio do penetrômetro digital Agrosta 100 Touchscreen (Agro-Technologie, Forges Les Eaux, França), com ponteira 25 (3,5 mm); padrões de cor $\{$ luminescência (\%) [L(D65)]\}; valor cromático no eixo vermelho-verde $[\mathrm{a}(\mathrm{D} 65)]$; valor cromático no eixo amarelo-azul $[\mathrm{b}(\mathrm{D} 65)]$, com colorímetro digital CM-508d (Konica Minolta Inc., Marunouchi, Chiyoda, Tokyo, Japão), em duas leituras na região equatorial das bagas; potencial de hidrogênio $(\mathrm{pH})$, com aparelho Marconi PA200 (Marconi Equipamentos para Laboratórios Ltda., Piracicaba, SP, Brasil); e teor de ácido tartárico, por titulação com hidróxido de sódio a $0,1 \mathrm{~N}$. Para estas avaliações, utilizaram-se as metodologias propostas pelas Normas analíticas (1985). Para cada parâmetro analisado, foram utilizadas 20 bagas (repetições), exceto para o $\mathrm{pH}$ (repetição única composta pelo suco de 20 bagas) e o ácido tartárico (três repetições). Os valores de cores foram corrigidos com padrão de luminescência $\left(L^{*}\right)=50$, valor cromático no eixo vermelho-verde $\left(\mathrm{a}^{*}\right)=-60$ (verde), e valor cromático no eixo amarelo-azul $\left(\mathrm{b}^{*}\right)=0$, tendo-se calculado o valor de variação a partir do verde puro, com $50 \%$ de luminescência no sistema CIE-LAB $\left(\Delta \mathrm{E}^{*}\right)$, pela fórmula: $\left.\left\{\left[(\mathrm{L}-50)^{2}\right]+[\mathrm{a}-(-60)]^{2}\right\}+\left[(\mathrm{b}-0)^{2}\right]\right\}^{0,5}$.

Para a instalação dos experimentos, as bagas dos diferentes genótipos foram previamente examinadas, com o auxílio de microscópio estereoscópico 40X (Carl Zeiss Microscopy GmbH, Göttingen, Alemanha), para verificar a integridade da epiderme e a ausência de posturas e de larvas de D. suzukii. Posteriormente, as bagas foram individualizadas em recipientes de plástico transparente $(120 \mathrm{~mL})$, fechados com parafilme (Bemis Company, Inc., Neenah, WI, EUA). Em seguida, fêmeas acasaladas de quatro dias de idade foram individualizadas nos recipientes que continham uma baga, por 24 horas, ao final das quais se procedeu à contagem do número de ovos, com auxílio de microscópio estereoscópico (40X).

Para cada genótipo, determinaram-se o percentual de frutos danificados (com puncturas) e o número médio de ovos por fruto, tendo-se realizado observações de orifícios na epiderme dos frutos (puncturas) e de filamentos (espiráculos) dos ovos que permanecem na parte externa dos frutos, por ocasião da oviposição. Após a contagem do número de ovos, os frutos foram individualizados em recipientes de plástico até a emergência dos insetos, verificada diariamente por um período de 25 dias, e o percentual de emergência e o tempo de desenvolvimento destes insetos foram determinados.

Para este ensaio, utilizou-se um delineamento experimental inteiramente causalizado, com 18 tratamentos (genótipos) e 30 repetições (bagas). Os dados de infestação (ovos por fruto) foram submetidos à análise de variância. As médias foram comparadas pelo teste Scott-Knott, a 1\% de probabilidade (SAS Institute, Cary, NC, EUA), e as cultivares foram classificadas, quanto à suscetibilidade, pelos grupos homogêneos deste mesmo teste. Por fim, realizou-se uma análise de correlação de Pearson entre as variáveis físico-químicas e o índice de infestação, cuja significância foi verificada pelo teste $t$ de Student, a $1 \%$ de probabilidade.

Para determinar se os danos causados pela mosca-das-frutas sul-americana, durante a oviposição, e os danos mecânicos pela ruptura da epiderme dos frutos podem favorecer a infestação pela $D$. suzukii, estabeleceram-se os seguintes tratamentos: Pafe, dano causado pela punctura de fêmeas de mosca-das-frutas sul-americana, previamente esterilizada com solução alimentar com $40 \mathrm{mg} \mathrm{L}^{-1}$ do i.a. novalurom Rimon 100 EC, (Milenia Agrociências S. A., Londrina, PR, Brasil) e atrativo alimentar BioAnastrepha a 5\% (BioControle Métodos de Controle de Pragas Ltda., Indaiatuba, SP, Brasil), à proporção de 1:20000 mL, conforme Machota Junior (2011); PAF, dano causado pela punctura de fêmeas de mosca-das-frutas sul-americana não esterilizadas e com presença de galerias formadas pelas larvas; DM, dano causado mecanicamente, com 20 incisões de alfinete entomológico de $0,25 \mathrm{~mm}$ de diâmetro, à profundidade de $2 \mathrm{~mm}$ em cada baga; SD, bagas sem danos (padrão de resistência); e MO, frutos de morango da cultivar 'Albion' (padrão de suscetibilidade). Nos tratamentos Pafe, PAF, DM e SD, foram utilizadas bagas de uva 'Italia', considerada resistente à $D$. suzukii, em ensaio a priori.

Para observação dos resultados do dano causado pela mosca-das-frutas sul-americana (tratamentos Pafe e PAF), cinco casais deste inseto, com 20 dias de idade, foram colocados em gaiolas de plástico $(500 \mathrm{~mL})$ com cinco bagas de uvas, pelo período de 24 horas. As posturas realizadas nas bagas, pela mosca-das-frutas sul-americana, foram contabilizadas com auxílio de microscópio estereoscópico de 40X (Carl Zeiss 
Microscopy GmbH, Göttingen, Alemanha), e a média de ovos por fruto foi obtida. Após 48 horas da retirada das fêmeas de moscas-das-frutas e realização dos danos mecânicos, as bagas de videiras e os morangos foram acondicionados em gaiolas de acrílico $(300 \mathrm{~mL})$, com cinco casais de D. suzukii, com quatro dias de idade, pelo período de 24 horas.

O número de ovos da $D$. suzukii, nas bagas uvas e nos morangos, foi determinado com o auxílio de microscópio estereoscópico de 40X, e o percentual de frutos danificados (puncturas) e o número médio de ovos por fruto, para cada tratamento, foram determinados por meio da visualização dos orifícios na epiderme dos frutos (puncturas) e de filamentos (espiráculos) dos ovos que permaneceram do lado de fora do fruto. Após a determinação do número de ovos postos, os frutos foram individualizados e mantidos em recipientes de plástico $(120 \mathrm{~mL})$, fechados com parafilme até a emergência dos insetos.

Utilizou-se o delineamento experimental inteiramente casualizado, com cinco tratamentos (tipos de danos) e 30 repetições (bagas). Pelo fato de os dados não apresentarem distribuição normal, em consequência das médias de valor zero, os dados de número de ovos e de adultos emergidos por fruto foram transformados por $(\mathrm{X}+0,1)^{0,5}$, em que $\mathrm{X}$ representou o valor observado. Em seguida, realizou-se a análise de variância, e as médias foram comparadas, pelo teste de Tukey, a $1 \%$ de probabilidade, com auxílio do programa estatístico SAS (SAS Institute, Cary, NC, EUA).

\section{Resultados e Discussão}

Na avaliação da suscetibilidade de genótipos de uva à D. suzukii, observaram-se posturas nas cultivares 'Benitaka' e 'Cabernet Sauvignon', 'BRS Lorena', 'BRS Isis', 'BRS Morena', 'BRS Nubia' e 'BRS Vitória', e na seleção CNPUV 1097-258 (Tabela 1). Os genótipos 'Benitaka', 'BRS Vitória' e 'BRS Morena' apresentaram mais de 2,4 ovos por baga e diferiram dos demais genótipos $\left(\mathrm{p}=0,0001 ; \chi^{2}=279,12\right.$; e graus de liberdade $=6$ ), compondo o primeiro grupo de suscetibilidade (GS1). Os demais genótipos infestados - 'BRS Isis', 'Carbenet Sauvignon', CNPUV 1097-258, 'BRS Nubia' e 'BRS Lorena' - ficaram no segundo grupo (GS2), que foi menos suscetível do que o primeiro grupo. Os genótipos não atacados ficaram em um terceiro grupo, considerado resistente (GR), que foram: as cultivares de V. labrusca - 'Concord', 'Isabel' e 'Niagara Rosada'; e as cultivares de $V$. vinifera - 'Italia', 'Itália Muscat', 'Moscato Bianco', 'Redglobe' e 'Italia Rubi' - e as seleções CNPUV 1060-4 e 20). Ioriatti et al. (2015) avaliaram a suscetibilidade de cultivares de videira, na Itália e no Oregon (EUA), e verificaram valores inferiores a duas posturas por baga nas cultivares de V. vinifera 'Chardonnay', 'Yellow Moscat', 'Traminer', 'Pinot Noir', 'Teroldego' e 'Schiava', valores que se aproximam aos obtidos no GS2 do presente trabalho.

Nenhum dos genótipos pertencentes a $V$. labrusca foi atacado pela $D$. suzukii. Este resultado foi importante, pois esta espécie de videira representa boa parte daquelas cultivadas no Sul do país (Camargo et al., 2011). As cultivares de V. labrusca são conhecidas por

Tabela 1. Média \pm erro-padrão do número de ovos por fruto, percentagem de frutos infestados, percentagem de emergência de insetos e tempo médio de desenvolvimento de drosófila-da-asa-manchada (Drosophila suzukii), em 18 genótipos de videira classificados em grupo suscetível 1 (GS1), grupo suscetível 2 (GS2) e grupo resistente (GR). Temperatura de $22 \pm 1{ }^{\circ} \mathrm{C}$, umidade relativa de $65 \pm 10 \%$ e fotófase de 12 horas $^{(1)}$.

\begin{tabular}{|c|c|c|c|c|}
\hline Genótipo & $\begin{array}{c}\text { Ovos por } \\
\text { baga }\end{array}$ & $\begin{array}{c}\text { Frutos } \\
\text { infestados } \\
(\%)\end{array}$ & $\begin{array}{c}\text { Emergência } \\
\text { de insetos } \\
(\%)\end{array}$ & $\begin{array}{r}\text { TMD } \\
\text { (dia) }\end{array}$ \\
\hline & \multicolumn{4}{|c|}{ Suscetíveis - GS1 } \\
\hline 'Benitaka' & $4,6 \pm 1,2 \mathrm{a}$ & 63,3 & 0,0 & - \\
\hline 'BRS Vitória' & $3,3 \pm 1,0 \mathrm{a}$ & 36,7 & 2,0 & $14,5(2)^{(2)}$ \\
\hline \multirow[t]{2}{*}{ 'BRS Morena' } & $2,4 \pm 0,8 \mathrm{a}$ & 36,7 & 5,7 & $19,8(4)$ \\
\hline & \multicolumn{4}{|c|}{ Suscetíveis - GS2 } \\
\hline 'BRS Isis' & $1,8 \pm 1,0 \mathrm{~b}$ & 20,0 & 5,4 & $15,0(3)$ \\
\hline 'Carbenet Sauvignon' & $1,1 \pm 1,0 \mathrm{~b}$ & 6,7 & 0,0 & - \\
\hline CNPUV 1097-258 & $0,5 \pm 0,8 \mathrm{~b}$ & 3,3 & 0,0 & - \\
\hline 'BRS Nubia' & $0,4 \pm 0,3 b$ & 13,3 & 0,0 & - \\
\hline \multirow[t]{2}{*}{ 'BRS Lorena' } & $0,2 \pm 0,1 \mathrm{~b}$ & 10,0 & 0,0 & - \\
\hline & \multicolumn{4}{|c|}{ Resistentes - GR } \\
\hline CNPUV 1060-4 & 0,0 & 0,0 & 0,0 & - \\
\hline 'Concord' & 0,0 & 0,0 & 0,0 & - \\
\hline 'Isabel' & 0,0 & 0,0 & 0,0 & - \\
\hline 'Italia' & 0,0 & 0,0 & 0,0 & - \\
\hline 'Itália Muscat' & 0,0 & 0,0 & 0,0 & - \\
\hline 'Italia Rubi' & 0,0 & 0,0 & 0,0 & - \\
\hline 'Moscato Bianco' & 0,0 & 0,0 & 0,0 & - \\
\hline 'Niagara Rosada' & 0,0 & 0,0 & 0,0 & - \\
\hline 'Redglobe' & 0,0 & 0,0 & 0,0 & - \\
\hline Seleção 20 & 0,0 & 0,0 & 0,0 & - \\
\hline
\end{tabular}

${ }^{(1)}$ Médias seguidas de letras iguais, nas colunas, pertencem ao mesmo grupo, pelo teste Scott-Knott, a 1\% de probabilidade. ${ }^{(2)}$ Valor entre parênteses representa o numero absoluto de insetos emergidos em cada cultivar. 
sua maior rusticidade, que lhes confere resistência ou tolerância a pragas e boa adaptabilidade a diferentes ambientes (Valadão et al., 2012; Bertin et al., 2013; Baronio et al., 2014). Entre os genótipos onde ocorreu oviposições, dois foram da espécie $V$. vinifera ('Benitaka' e 'Cabernet Sauvignon'), e os demais foram cultivares desenvolvidas pelo programa de melhoramento da Embrapa.

Embora em alguns genótipos tenha ocorrido elevado número de posturas por fruto, o que pode ser considerado como um fator de suscetibilidade, a percentagem de adultos emergidos por baga foi baixa (Tabela 1). A emergência dos insetos ocorreu apenas nos genótipos 'BRS Vitória', 'BRS Morena' e 'BRS Isis'. Os resultados de emergência da $D$. suzukii, obtidos no presente estudo, foram inferiores aos observados por Ioriatti et al. (2015), que registraram emergência de até $36,5 \%$ em $V$. vinifera 'Traminer'. No entanto, o percentual de frutos infestados com ovos foi menor do que $30 \%$, o que indica menor suscetibilidade dos genótipos testados no presente trabalho, em comparação a $V$. vinifera 'Traminer'. O tempo médio de desenvolvimento da $D$. suzukii nas cultivares 'BRS Vitória' e 'BRS Isis' foi similar ao observado em morango 'Albion' (14 dias). Tochen et al. (2014) também obtiveram resultados similares em cereja e mirtilo, a $22^{\circ} \mathrm{C}$. A cultivar de uva 'BRS Morena' propiciou maior duração do desenvolvimento da $D$. suzukii do que o morangueiro e as demais cultivares de uva. Nos dois casos, o tempo de desenvolvimento foi maior do que o obtido por Emiljanowicz et al. (2014), que foi de 12,8 dias sob dieta artificial a $22^{\circ} \mathrm{C}$. Assim, observou-se que, pelo menos uma das três cultivares que permitiram o completo desenvolvimento do inseto limita-o a um maior período de desenvolvimento, o que implica, provavelmente, número menor de gerações por ano, com consequente redução da pressão populacional da praga, em relação aos demais genótipos.

Observou-se que a postura foi realizada externamente à baga, nas cultivares 'Concord' (quatro bagas com 14 ovos), 'Isabel' (duas bagas com três ovos), 'Niagara Rosada' (três bagas e três ovos), 'Italia' (duas bagas e três ovos), 'Redglobe' (duas bagas e dois ovos) e Seleção 20 (quatro bagas e quatro ovos). Nestes seis genótipos, os ovos não deram origem a larvas e apresentaram aspecto de desidratação nas primeiras 24 horas após inicio da exposição dos frutos às moscas.
As características físico-químicas, avaliadas nas bagas dos genótipos de videira, apresentaram correlações não significativas com o índice de oviposição, o que impediu o estabelecimento de um modelo matemático explicativo da variável suscetibilidade em função da resistência da epiderme, cor, $\mathrm{pH}$ e teor de ácido tartárico (Tabela 2).

Alguns fatores como a evolução da maturação de uvas podem estar relacionados ao início do ataque e à aceitação hospedeira pela mosca, conforme evidenciado por Ioriatti et al. (2015). Estes autores observaram aumento gradativo do ataque da $D$. suzukii, associado à maturação de cultivares de videira e, principalmente, à diminuição da resistência à penetração da epiderme e de componentes ácidos, além da elevação dos teores de sólidos solúveis. Outros trabalhos sobre videira também mostraram que a resistência à penetração foi o principal fator ligado à suscetibilidade de uvas à D. suzukii, que responderam de forma negativa à oviposição (Lee et al., 2011; Burrack et al., 2013; Kinjo et al., 2013; Ioriatti et al., 2015). Porém, no atual trabalho, não se obteve correlação significativa entre esses fatores e o índice de ataque da D. suzukii. Isto ocorreu porque não houve correlação à evolução da maturação, e houve consequente perda de firmeza das bagas das cultivares com os índices de infestação. Este fato mostra a presença de fatores ainda desconhecidos que podem influenciar a escolha do hospedeiro.

Ademais, além de as cultivares de $V$. vinifera serem em geral mais suscetíveis a pragas e doenças do que as cultivares de $V$. labrusca, os programas de melhoramento vegetal, ao buscarem melhorias em parâmetros de qualidade e produtividade de plantas podem, em alguns casos, gerar uma redução direta de características que conferem rusticidade, resistência ou tolerância a pragas. Assim, a busca por resistência a doenças, principalmente fúngicas, pode estar relacionada ao aumento de suscetibilidade a outras pragas, como o ocorrido com o genótipo 'BRS Isis', que é resistente ao míldio (Ritschel et al., 2013) e apresentou o segundo maior índice de infestação pela D. suzukii (Tabela 1).

O experimento para avaliar a interação entre os danos da mosca-das-frutas sul-americana esterilizada (PAFE), não esterilizada (PAF) e os danos mecânicos (DM), em bagas de uva $V$. vinifera 'Italia', não apresentou valores expressivos de oviposição e de emergência de insetos (Figura 1). Em comparação, a testemunha 
de suscetibilidade, o morango 'Albion', apresentou $96,7 \%$ dos frutos com ovos e $63,3 \%$ de emergência. Observaram-se diferenças quanto ao número de ovos e adultos do inseto emergidos por fruto, em morango, em comparação aos demais tratamentos, $\left(\mathrm{F}_{(4,145)}=120,99\right.$, $\mathrm{p}=0,0001 ; \mathrm{F}_{(4,145)}=28,46, \mathrm{p}=0,0001$, respectivamente). Isto mostra que os insetos usados no atual experimento se encontravam aptos à infestação e à reprodução, além de mostrar menor adequação hospedeira de uvas íntegras ou danificadas à $D$. suzukii, em comparação ao morango. A duração do desenvolvimento no morango foi de 14,4 dias, enquanto em bagas de uva 'Italia' ovipositadas pela mosca-das-frutas sul-americana não estéril foi de 19,5 dias.

O baixo potencial da videira, como hospedeiro da D. suzukii isoladamente e em comparação com outros hospedeiros, também foi evidenciada por outros autores (Maiguashca et al., 2010; Lee et al., 2011; Bellamy et al., 2013). No entanto, danos causados por pássaros, granizo, doenças ou rachaduras, em decorrência do excesso de umidade e de chuva, podem ser um agravante para o ataque da $D$. suzukii em videira (Ioriatti et al., 2015).

Não se observou incremento da infestação da $D$. suzukii em $V$. vinifera 'Italia', após a epiderme ter sido mecanicamente perfurada, tanto de forma artificial como pela punctura de oviposição ou presença de larvas da mosca-das-frutas sul-americana. Estes resultados reforçam a hipótese de que a tolerância ao ataque da $D$. suzukii, pela uva 'Italia', não está diretamente relacionada à resistência da epiderme. Nesse caso, outros fatores ou sinais químicos podem ser determinantes para a aceitação hospedeira. Assim, considera-se que $D$.suzukiinãoutiliza obrigatoriamente danos na epiderme de bagas de uva 'Italia', causadas pelo ataque da mosca-das-frutas sul-americana ou danos mecânicos, para sua oviposição. A razão para tal fenômeno poderia ser explicada por duas hipóteses: os danos causados superam a barreira física da epiderme, porém, produzem algum efeito ou

Tabela 2. Média \pm erro-padrão da resistência da epiderme, coloração, $\mathrm{pH}$ e ácido tartárico de 18 genótipos de videira, avaliados quanto à suscetibilidade à drosófila-da-asa-manchada (Drosophila suzukii) em laboratório.

\begin{tabular}{|c|c|c|c|c|}
\hline Genótipo & $\begin{array}{l}\text { Resistência da epiderme }^{(1)} \\
\text { (Durofel } 25 \text { unit) }\end{array}$ & Coloração $\left(\Delta \mathrm{E}^{*}\right)^{(1)}$ & $\mathrm{pH}^{(2)}$ & $\begin{array}{l}\text { Ácido tartárico }{ }^{(3)} \\
\left(\mathrm{g} 100 \mathrm{~mL}^{-1}\right)\end{array}$ \\
\hline \multicolumn{5}{|l|}{ Vitis labrusca } \\
\hline 'Concord' & $18,6 \pm 0,6$ & $62,86 \pm 0,41$ & 3,52 & $1,76 \pm 0,01$ \\
\hline 'Isabel' & $49,9 \pm 0,7$ & $63,39 \pm 0,24$ & 3,08 & $1,94 \pm 0,01$ \\
\hline 'Niagara Rosada' & $37,2 \pm 0,6$ & $65,74 \pm 0,22$ & 3,64 & $1,21 \pm 0,04$ \\
\hline \multicolumn{5}{|l|}{ Vitis vinifera } \\
\hline 'Benitaka' & $46,1 \pm 0,7$ & $70,10 \pm 0,32$ & 3,73 & $1,04 \pm 0,01$ \\
\hline 'Cabernet Sauvignon' & $52,4 \pm 0,8$ & $62,65 \pm 0,27$ & 3,54 & $2,09 \pm 0,03$ \\
\hline 'Italia' & $42,4 \pm 0,6$ & $59,60 \pm 0,40$ & 3,14 & $1,52 \pm 0,05$ \\
\hline 'Italia Muscat' & $39,1 \pm 0,9$ & $62,59 \pm 0,89$ & 3,42 & $1,51 \pm 0,09$ \\
\hline 'Italia Rub'i & $43,9 \pm 0,6$ & $82,38 \pm 0,33$ & 3,37 & $1,40 \pm 0,05$ \\
\hline 'Moscato Bianco' & $44,8 \pm 0,8$ & $59,09 \pm 0,25$ & 3,21 & $2,51 \pm 0,07$ \\
\hline 'Redglobe' & $55,7 \pm 1,2$ & $65,74 \pm 0,36$ & 3,64 & $1,21 \pm 0,03$ \\
\hline \multicolumn{5}{|l|}{ Melhoradas } \\
\hline 'BRS Isis' & $71,9 \pm 1,4$ & $67,71 \pm 0,54$ & 3,88 & $0,77 \pm 0,03$ \\
\hline 'BRS Lorena' & $37,7 \pm 0,6$ & $59,76 \pm 0,60$ & 3,56 & $1,34 \pm 0,04$ \\
\hline 'BRS Morena' & $65,5 \pm 0,8$ & $65,35 \pm 0,20$ & 3,73 & $1,08 \pm 0,03$ \\
\hline 'BRS Nubia' & $41,9 \pm 0,6$ & $63,49 \pm 0,38$ & 3,54 & $1,32 \pm 0,02$ \\
\hline 'BRS Vitória' & $58,7 \pm 1,3$ & $64,52 \pm 0,27$ & 3,60 & $1,11 \pm 0,03$ \\
\hline \multicolumn{5}{|l|}{ Seleções da Embrapa } \\
\hline CNPUV 1060-4 & $66,2 \pm 0,8$ & $66,74 \pm 0,54$ & 3,69 & $1,01 \pm 0,03$ \\
\hline CNPUV 1097-258 & $46,1 \pm 0,7$ & $59,50 \pm 0,35$ & 3,62 & $0,92 \pm 0,02$ \\
\hline Seleção 20 & $37,1 \pm 1,1$ & $64,47 \pm 0,43$ & 3,60 & $1,30 \pm 0,02$ \\
\hline Correlação de Pearson $^{(4)}$ & $0,40^{\text {ns }}$ & $0,48^{\text {ns }}$ & $0,48^{\text {ns }}$ & $-0,38^{\text {ns }}$ \\
\hline
\end{tabular}


composto "não estimulante" ou repelente à D. suzukii; e esta cultivar possui algum fator de resistência que não está relacionado à espessura e resistência da epiderme.

Keesey et al. (2015) sugerem que respostas olfativas são responsáveis pela troca de nicho da $D$. suzukii de frutas em deterioração para frutas sadias. Isto mostra que a $D$. suzukii apresenta reduzida resposta neurológica a compostos fermentados (como hexonoato etílico e metílico), geralmente ligados à deterioração do tecido vegetal, em comparação à mosca Drosophila melanogaster (Meigen, 1830), porém mostra elevada resposta fisiológica e comportamental a compostos produzidos nas folhas de morangueiro, em resposta fisiológica ao amadurecimento dos frutos. Essas respostas fisiológicas reforçam a segunda hipótese levantada no presente trabalho, de que a resistência ou preferencia hospedeira da D. suzukii não está unicamente relacionada à espessura e resistência da epiderme.

Assim, a videira é considerada um hospedeiro não adequado ao desenvolvimento da $D$. suzukii, e

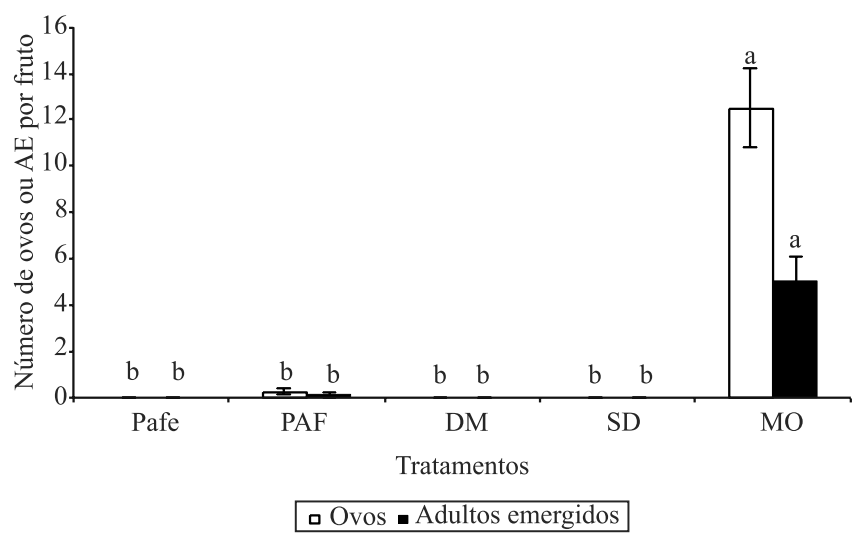

Figura 1. Número de ovos e de adultos emergidos (AE) de drosófila-da-asa-manchada (Drosophila suzukii), em bagas de Vitis vinifera 'Italia' submetidas a diferentes tipos de danos: Pafe, previamente ovipositadas por Anastrepha fraterculus estéril; PAF, previamente ovipositadas por $A$. fraterculus não estéril; DM, com dano mecânico feito com puncturas de alfinete; SD, sem danos mecânicos; e MO, testemunha (morango 'Albion', sem danos mecânicos). Colunas seguidas de letras iguais não diferem entre si, pelo teste de Tukey, a 1\% de probabilidade. as puncturas da mosca-das-frutas sul-americana não aumentam os índices de infestação da praga, o que resulta em baixo potencial de perdas econômicas que poderia ser causado pelo ataque do inseto à cultura.

\section{Conclusões}

1. Genótipos de videiras apresentam reduzida suscetibilidade ao ataque de drosófila-da-asa-manchada (Drosophila suzukii).

2. As bagas das cultivares 'Benitaka', 'BRS Vitória', 'BRS Morena', 'BRS Isis', 'Carbenet Sauvignon', , 'BRS Nubia' e 'BRS Lorena' e o CNPUV 1097-258 são suscetíveis a $D$. suzukii.

3. As bagas dos genótipos 'Concord', 'Isabel', 'Itália', 'Itália Muscat', 'Italia Rubi', 'Moscato Bianco', 'Niagara Rosada', 'Redglobe', Seleção 20 e CNPUV 1060-4 são resistentes ao ataque de $D$. suzukii.

4. Danos mecânicos ou causados pela oviposição prévia de $A$. fraterculus não influenciam a suscetibilidade de bagas de uva 'Italia' ao ataque de D. suzukii.

\section{Agradecimentos}

Ao Conselho Nacional de Desenvolvimento Científico e Tecnológico (CNPq), pelo apoio financeiro; aos Srs. Sandro Daniel Nörnberg, Tiago Scheunemann, Ricardo Braun Marangon e Daniel Bernardi, pelo auxílio na criação de manutenção e discussões sobre o trabalho; e à Equipe do Laboratório de Qualidade Pós-colheita, da Embrapa Uva e Vinho, pelas análises realizadas.

\section{Referências}

BARONIO, C.A.; ANDZEIEWSKI, S.; CUNHA, U.S. da; BOTTON, M. Biologia e tabela de vida de fertilidade do pulgão-preto em cultivares de videira. Pesquisa Agropecuária Brasileira, v.49, p.665-672, 2014. DOI: 10.1590/S0100-204X2014000900002.

BELLAMY, D.E.; SISTERSON, M.S.; WALSE, S.S. Quantifying host potentials: indexing postharvest fresh fruits for spotted wing drosophila, Drosophila suzukii. Plos One, v.8, p.e61227, 2013. DOI: 10.1371/journal.pone.0061227.

BERTIN, A.; BORTOLI, L.C.; BOTTON, M.; PARRA, J.R.P. Host plant effects on the development, survival, and reproduction of Dysmicoccus brevipes (Hemiptera: Pseudococcidae) on grapevines. Annals of the Entomological Society of America, v.106, p.604-609, 2013. DOI: 10.1603/AN13030. 
BURRACK, H.J.; FERNANDEZ, G.E.; SPIVEY, T.; KRAUS, D.A. Variation in selection and utilization of host crops in the field and laboratory by Drosophila suzukii Matsumara (Diptera: Drosophilidae), an invasive frugivore. Pest Management Science, v.69, p.1173-1180, 2013. DOI: 10.1002/ps.3489.

CAMARGO, U.A.; TONIETTO, J.; HOFFMANN, A. Progressos na viticultura brasileira. Revista Brasileira de Fruticultura, v.33, p.144-149, 2011. DOI: 10.1590/S0100-29452011000500017.

CINI, A.; ANFORA, G.; ESCUDERO-COLOMAR, L.A.; GRASSI, A.; SANTOSUOSSO, U.; SELJAK, G.; PAPINI, A. Tracking the invasion of the alien fruit pest Drosophila suzukii in Europe. Journal of Pest Science, v.87, p.559-566, 2014. DOI: 10.1007/s10340-014-0617-z.

CINI, A.; IORIATTI, C.; ANFORA, G. A review of the invasion of Drosophila suzukii in Europe and a draft research agenda for integrated pest management. Bulletin of Insectology, v.65, p.149-160, 2012.

DEPRÁ, M.; POPPE, J.L.; SCHMITZ, H.J.; DE TONI, D.C.; VALENTE, V.L.S. The first records of the invasive pest Drosophila suzukii in the South American continent. Journal of Pest Science, v.87, p.379-383, 2014. DOI: 10.1007/s10340-014-0591-5.

EMILJANOWICZ, L.M.; RYAN, G.R.; LANGILLE, A.; NEWMAN, J. Development, reproductive output and population growth of the fruit fly pest Drosophila suzukii (Diptera: Drosophilidae) on artificial diet. Journal of Economic Entomology, v.107, p.1392-1398, 2014. DOI: 10.1603/EC13504.

IORIATTI, C.; WALTON, V.; DALTON, D.; ANFORA, G.; GRASSI, A.; MAISTRI, S.; MAZZONI, V. Drosophila suzukii (Diptera: Drosophilidae) and its potential impact to wine grapes during harvest in two cool climate wine grape production regions. Journal of Economic Entomology, v.108, p.1148-1155, 2015. DOI: $10.1093 /$ jee/tov042.

KEESEY, I.W.; KNADEN, M.; HANSSON, B.S. Olfactory specialization in Drosophila suzukii supports an ecological shift in host preference from rotten to fresh fruit. Journal of Chemical Ecology, v.41,p.121-128, 2015. DOI: 10.1007/s10886-015-0544-3.

KINJO, H.; KUNIMI, Y.; BAN, T.; NAKAI, M. Oviposition efficacy of Drosophila suzukii (Diptera: Drosophilidae) on different cultivars of blueberry. Journal of Economic Entomology, v.106, p.1767-1771, 2013. DOI: 10.1603/EC12505.

LEE, J.C.; BRUCK, D.J.; CURRY, H.; EDWARDS, D.; HAVILAND, D.R.; VAN STEENWIK, R.A.; YORGEY, B.M. The susceptibility of small fruits and cherries to the spotted-wing drosophila, Drosophila suzukii. Pest Management Science, v.67, p.1358-1367, 2011. DOI: 10.1002/ps.2225.

MACHOTA JUNIOR, R. Associação entre injúrias causadas por fêmeas de Anastrepha fraterculus (Wied.) (Diptera: Tephritidae) e patógenos responsáveis por podridões em cachos na cultura da videira em laboratório. 2011. 68p. Dissertação (Mestrado) Universidade Federal de Pelotas, Pelotas, RS.

MACHOTA JUNIOR, R.; BORTOLI, L.C.; BOTTON, M.; GRÜTZMACHER, A.D. Fungi that cause rot in bunches of grape identified in adult fruit flies (Anastrepha fraterculus) (Diptera: Tephritidae). Chilean Journal of Agricultural Research, v.73, p.196-201, 2013. DOI: 10.4067/S0718-58392013000200018.

MAIGUASHCA, F.; FERGUSSON, H.; BAHDER, B.; BROOKS, T.; O'NEAL, S.; WALSH, D. SWD ovipositing on grapes in laboratory; partial maggot survival inconclusive. 2010. Available at: $<$ http://ipm.wsu.edu/small/pdf/NoChoiceSWDonGrapesAug28. pdf $>$. Accessed on: 9 Feb. 2015.

NORMAS analíticas: métodos químicos e físicos para análises de alimentos. São Paulo: Instituto Adolfo Lutz, 1985. 533p.

RITSCHEL, P.S.; MAIA, J.D.G.; CAMARGO, U.A.; SOUZA, R.T. de; FAJARDO, T.V.M.; NAVES, R. de L.; GIRARDI, C.L. BRS Isis: nova cultivar de uva de mesa vermelha, sem sementes e tolerante ao míldio. Bento Gonçalves: Embrapa Uva e Vinho, 2013. 20p. (Embrapa Uva e Vinho. Comunicado técnico, 143).

SAGUEZ, J.; LASNIER, J.; VINCENT, C. First record of Drosophila suzukii in Quebec vineyards. Journal Internacional des Science de la Vigne et du Vin, v.47, p.69-72, 2013.

SANTOS, R.S.S. dos. Drosophila suzukii (Matsumura, 1931) (Diptera: Drosophilidae) atacando frutos de morangueiro no Brasil. Enciclopédia Biosfera, v.10, p.4005-4011, 2014.

TOCHEN, S.; DALTON, D.T.; WIMAN, N.; HAMM, C.; SHEARER, P.W. Temperature-related development and population parameters for Drosophila suzukii (Diptera: Drosophilidae) on cherry and blueberry. Environmental Entomology, v.43, p.501-510, 2014. DOI: 10.1603/EN13200.

VALADÃO, G.S.; VIEIRA, M.R.; PIGARI, S.A.A.; TABET, V.G.; SILVA, A.C. da. Resistência de cultivares de videira ao ácaro-rajado Tetranychus urticae na região de Jales, Estado de São Paulo. Revista Brasileira de Fruticultura, v.34, p.1051-1058, 2012. DOI: 10.1590/S0100-29452012000400011.

VAN TIMMEREN, S.; ISAACS, R. Drosophila suzukii in Michigan vineyards, and the first report of Zaprionus indianus from this region. Journal of Applied Entomology, v.138, p.519-527, 2014. DOI: $10.1111 /$ jen.12113.

VILELA, R.C.; MORI, L. The invasive spotted-wing drosophila (Diptera, Drosophilidae) has been found in the city of São Paulo (Brazil). Revista Brasileira de Entomologia, v.58, p.371-375, 2014. DOI: $10.1590 / \mathrm{S} 0085-56262014000400004$.

ZART, M.; BOTTON, M.; FERNANDES, O.A. Injúrias causadas por mosca-das-frutas sul-americana em cultivares de videira. Bragantia, v.70, p.64-71, 2011. DOI: 10.1590/ S0006-87052011000100011.

$\overline{\text { Recebido em } 9 \text { de junho de } 2015 \text { e aprovado em } 9 \text { de setembro de } 2015}$ 